\title{
Variação temporal das taxas de vacinação contra a brucelose bovina no Sudoeste
}

\section{Goiano}

Temporal variation of vaccination rates against bovine brucellosis in the Southeast of Goias State Variación temporal de las tasas de vacunación contra la brucelosis bovina en el Sureste Goiano

Rafaela Assis Oliveira ORCID: https://orcid.org/0000-0001-6367-3921 Universidade Federal de Jataí, Brasil E-mail: rafaela_assis97@ hotmail.com

Carolina de Alvarenga Cruz ORCID: https://orcid.org/0000-0002-1623-8932 Universidade Estadual Paulista, Brasil E-mail: carol_a_cruz@yahoo.com.br

Luis Antonio Mathias

ORCID: https://orcid.org/0000-0001-7282-3071 Universidade Estadual Paulista, Brasil E-mail: la.mathias@unesp.br

David Attuy Vey da Silva ORCID: https://orcid.org/0000-0002-9992-7513 German Federal Institute for Risk Assessment, Department of Biological Safety, Germany E-mail: attuy.david@gmail.com

Maria Fabiana Cipolini Galarza ORCID: https://orcid.org/0000-0003-1152-8970 Universidad Nacional del Nordeste, Argentina E-mail: fabicipolini@gmail.com

Clayton Bernardinelli Gitti ORCID: https://orcid.org/0000-0001-7435-7561 Universidade Federal Rural do Rio de Janeiro, Brasil E-mail: cbgitti@yahoo.com.br

Alana Flávia Romani ORCID: https://orcid.org/0000-0002-8138-408X Universidade Federal de Jataí, Brasil E-mail: alana_romani@ufj.edu.br

Andreia Vitor Couto do Amaral ORCID: https://orcid.org/0000-0001-6406-2372 Universidade Federal de Jataí, Brasil E-mail: andreiavcvet@ufj.edu.br

Dirceu Guilherme de Souza Ramos ORCID: https://orcid.org/0000-0001-9603-6638 Universidade Federal de Jataí, Brasil E-mail: dguilherme@ufj.edu.br

Klaus Casaro Saturnino ORCID: https://orcid.org/0000-0001-8493-8669 Universidade Federal de Jataí, Brasil E-mail: klaus.sat@ufj.edu.br Ísis Assis Braga

ORCID: https://orcid.org/0000-0001-5713-4833 Centro Universitário de Mineiros, Brasil E-mail: isis@unifimes.edu.br

Eric Matheus Nascimento de Paula ORCID: https://orcid.org/0000-0002-5948-1860

Centro Universitário de Mineiros, Brasil E-mail: ericmateus@unifimes.edu.br

Daniel Bartoli de Sousa

ORCID: https://orcid.org/0000-0003-3209-7911 Universidade Federal de Jataí, Brasil

E-mail: daniel_bartoli_sousa@ufj.edu.br

Raphaella Barbosa Meirelles-Bartoli ORCID: https://orcid.org/0000-0002-7147-5711 Universidade Federal de Jataí, Brasil E-mail: raphaella@ufj.edu.br 


\title{
Resumo
}

A brucelose bovina é uma enfermidade infecciosa, crônica e de notificação obrigatória, responsável por inúmeras perdas econômicas e estabelecimento de barreiras sanitárias. O estudo foi realizado com o intuito de analisar a tendência de longo prazo das taxas de vacinação na microrregião do Sudoeste Goiano, no período de 2015 a 2020. Calculou-se as taxas de vacinação contra a brucelose bovina para, posteriormente, realizar os cálculos de tendência temporal de vacinação e suas análises. Os dados de vacinação do rebanho bovino, do período em questão, foram fornecidos pela Agência Goiana de Defesa Agropecuária (Agrodefesa), Goiânia-Go. No Sudoeste Goiano, a taxa de vacinação contra a brucelose foi de $59 \%$ no primeiro semestre de 2015 , e de $35 \%$ no segundo semestre do mesmo ano. Em 2017, no primeiro semestre, a taxa foi de 54\%, e de $29 \%$ no segundo semestre. No primeiro semestre de 2018 , a taxa de vacinação observada foi de $48 \%$, e de $25 \%$ no segundo semestre. No primeiro e segundo semestres de 2019 , foram observadas, respectivamente, taxas de vacinação de $58 \%$ e $29 \%$. Por fim, no primeiro semestre de 2020 , observou-se uma taxa de 58\%. A média das taxas de vacinação nos semestres avaliados foi de $44 \%$. A menor taxa de vacinação foi observada em Chapadão do Céu (8\%) no segundo semestre de 2017, com taxa de variação semestral de - 15,13\% ( $\mathrm{IC}_{95 \%}$ - 28,7 a 1). Santa Rita do Araguaia apresentou as duas maiores taxas de vacinação, a primeira, $117 \%$, observada no primeiro semestre de 2020, com taxa de variação semestral de - 8,57\% (IC $95 \%$ - 20,2 a 4,8), e a segunda, $92 \%$, observada no primeiro semestre de 2017, com taxa de variação semestral de - 8,57\% (IC $95 \%$ - 20,2 a 4,8). As tendências de longo prazo das taxas de vacinação foram de estabilidade na maioria dos municípios avaliados. Uma vez que grande parte das taxas foram inferiores a $70 \%$ em todos os anos avaliados, essa estabilidade se torna desfavorável. Os resultados nos sugerem pouca evolução no programa de vacinação contra a brucelose bovina no Sudoeste Goiano o que pode influenciar negativamente a prevalência da doença na região.

Palavras-chave: Defesa agropecuária; Profilaxia; Zoonoses.

\begin{abstract}
The bovine brucellosis is an infectious disease, as well as a chronic disorder of compulsory notification, responsible for several losses and the institution of sanitary barriers. The study had the purpose of analyzing the tendency the long-term vaccination in the Southwestern micro-region of Goiás, in between the years 2015 and 2020. The rates of vaccination against bovine brucellosis were calculated, so, later, the time trends of the vaccination could be calculated and analyzed. The data about the vaccination of the cattle herd, during the relevant period were garnered from the Goiana Agency of Agricultural Defence (Agrodefesa). In the Southwest of Goiás, the vaccination rates against brucellosis was $59 \%$ on the first semester of 2015 and $35 \%$ on the second semester of the same year. In 2017, it was $54 \%$ in the first semester and $29 \%$ in the second. On the first semester of 2018 , the rates observed was $48 \%$ and $25 \%$ on the second. On the first and second semesters of 2019, they were, respectively, $58 \%$ and $29 \%$. Finally, on the first semester of 2020 , it reached $58 \%$. The average of the vaccination rates on the aforementioned semesters was $44 \%$. The area with the least vaccination amongst those studied was Chapadão do Céu (8\%), on the second semester of 2017, and variation rates between semesters were $15.13 \%$ ( IC $_{95 \%}-28.7$ a 1). Santa Rita do Araguaia had the two highest vaccination rates, $117 \%$ - the highest, on the first semester of 2020 with variation rates between semesters of $8,57 \%$ (IC $95 \%$ - 20,2 a 4,8) - and 92\% - the second highest, on the first semester of 2017 and variation rates between semesters of $8,57 \%$ ( $\mathrm{IC}_{95 \%}$ - 20,2 a 4,8). The long-term time trends were stable on most of the assessed municipalities. Considering that most of the rates were lower than $70 \%$, on all the relevant semesters, the stability is a negative development. The results suggest little evolution on the vaccination program against bovine brucellosis on the Goiano Southwest, which could affect the prevalence of the disease on the micro-region in a negative fashion.
\end{abstract}

Keywords: Agricultural defense; Prophylaxis; Zoonosis.

\section{Resumen}

La brucelosis bovina es una enfermedad infecciosa, crónica y de declaración obligatoria, responsable por numerosas pérdidas económicas y el establecimiento de barreras sanitarias. El estudio se realizó con el objetivo de analizar la tendencia a largo plazo de las tasas de vacunación en la microrregión del suroeste de Goiás, en el período de 2015 a 2020. Se calcularon las tasas de vacunación contra la brucelosis bovina, para posteriormente realizar los cálculos de Tendencia temporal de la vacunación y sus análisis. Los datos de vacunación del rebaño bovino, del período en cuestión, fueron proporcionados por la Agencia de Defensa Agrícola Goiana (Agrodefesa). En el suroeste de Goiás, la tasa de vacunación contra la brucelosis fue de $59 \%$ en el primer semestre de 2015 y de $35 \%$ en el segundo semestre del mismo año. En 2017, en el primer semestre, la tasa fue de $54 \%$ y de $29 \%$ en el segundo semestre. En el primer semestre de 2018, la tasa de vacunación observada fue de $48 \%$ y de $25 \%$ en el segundo semestre. En el primer y segundo semestre de 2019 se observaron tasas de vacunación de 58\% y 29\%, respectivamente. Finalmente, en el primer semestre de 2020 se observó una tasa de $58 \%$. La tasa de vacunación promedio en los semestres evaluados fue de 44\%. La tasa de vacunación más baja se observó en Chapadão do Céu (8\%), en el segundo semestre de 2017, con una tasa de variación semestral de - 15,13\% (IC 95\% - 28,7 a 1). Santa Rita do Araguaia presentó las dos tasas de vacunación más altas, la primera, $117 \%$, observada en el primer semestre de 2020, con una tasa de variación semestral de - 8,57\% (IC 95\% - 20,2 a 4,8), y el segundo, 92\%, observado en el primer semestre de 2017, con una tasa de variación semestral de - 8,57\% (IC de 95\% - 20,2 a 4,8). Las tendencias a largo plazo en las tasas de vacunación se mantuvieron estables en la mayoría de los municipios evaluados. Dado que la mayoría de las tasas fueron inferiores al 
$70 \%$, en todos los años evaluados, su estabilidad se vuelve desfavorable. Los resultados sugieren pocos avances en el programa de vacunación contra la brucelosis bovina en el suroeste de Goiás, lo que puede tener una influencia negativa en la prevalencia de la enfermedad en la región.

Palabras clave: Defensa agrícola; Profilaxis; Zoonosis.

\section{Introdução}

Considerada endêmica em todo o Brasil a brucelose bovina não tem sua ocorrência associada ao sistema de produção adotado, podendo ser identificada em qualquer tipo de propriedade. No entanto, o impacto econômico é maior no sistema de produção leiteiro. Isso porque os prejuízos estão relacionados a casos de aborto, a casos de infertilidade, além dos custos com reposição de fêmeas e assistência veterinária, causando, consequentemente, redução da produção de leite (Megid et al., 2016; Santos et al., 2017).

A ineficácia das medidas isoladas adotadas pelos estados brasileiros em anos anteriores, além da necessidade de padronização das ações de controle e profilaxia influenciaram para que, em 2001, o MAPA implementasse o Programa Nacional de Controle e Erradicação de Brucelose e Tuberculose Animal (PNCEBT), com o intuito de reduzir a incidência e prevalência de ambas enfermidades, além de aumentar o número de propriedades certificadas como livres e, consequentemente, contribuir para a produção e comercialização de produtos seguros e livres de contaminação (Brasil, 2006).

Desde sua implementação, o PNCEBT adota medidas obrigatórias e de adesão voluntária. É exigido aos produtores de gado, a vacinação de fêmeas bovinas e bubalinas de três a 8 meses com a amostra da vacina B19, ou a vacinação de fêmeas de qualquer idade com a amostra RB51. A eutanásia dos reagentes positivos e o controle do trânsito de animais por meio da Guia de Trânsito Animal (GTA), também são consideradas medidas obrigatórias. Opcionalmente, cabe ao produtor realizar a certificação da sua propriedade como livre da brucelose bovina (Brasil, 2017).

A Austrália, um dos países que obtiveram sucesso no controle da brucelose bovina, buscou adotar em seu programa de controle, a vacinação como medida primária contra a enfermidade, por exigir menor investimento e apresentar bons resultados (Austrália, 2019). Os Estados Unidos, outro país livre da brucelose, alcançou esse status em 2000, a partir da investigação epidemiológica e vacinação dos rebanhos (Ragan, 2002). A comparação entre estudos epidemiológicos de estados brasileiros também revelou a eficácia dessa medida na redução da prevalência da brucelose no país. No Brasil, desde dezembro de 2003, a vacinação das fêmeas é obrigatória para todos os estados, e a administração da vacina é de responsabilidade dos médicos veterinários cadastrados no serviço oficial de defesa sanitária animal. Santa Catarina é o estado brasileiro com menor índice de prevalência (0,9\%), cuja vacinação com a amostra B19 tem sido proibida desde 2004 (Brasil, 2006; Ferreira Neto et al., 2016).

Estudos transversais foram realizados nos anos de 1977 e 1982 nos estados brasileiros, refletindo a situação epidemiológica da enfermidade, de forma a orientar as ações de controle e profilaxia mais adequadas para cada situação (Leal Filho et al., 2016). Apesar da relevância, poucos estudos bem planejados e de vasta abrangência foram executados, afetando o conhecimento da real condição em que a doença se encontra e o desenvolvimento do programa (Poester et al., 2009).

No Estado de Goiás, o primeiro estudo epidemiológico com planejamento e precisão amostral foi realizado em 2009. Segundo Rocha et al. (2009), a prevalência de animais soropositivos em Goiás foi de 3,0\% nesse mesmo ano. Esses valores são inferiores aos encontrados no inquérito nacional realizado na década de 1970 (Brasil, 1977) e aos relatados por Santana e Veiga (1982), que estimaram, respectivamente, prevalências de 11,6\% e 9,0\% no estado. Baixas taxas de prevalência são esperadas nesses estudos, uma vez que a ausência de um banco de dados para organização das informações epidemiológicas referentes a brucelose, pode ter influenciado na geração de informações não fidedignas e dados de prevalência não condizentes com a realidade na época. 
No estudo mais recente (Rocha et al., 2009), Goiás foi dividido em três diferentes circuitos pecuários, conforme proposto pela Agrodefesa. Essa divisão foi embasada na aptidão do rebanho bovino predominante em cada região, como rebanho de corte, leite e misto. O Sudoeste Goiano foi designado como circuito 3, e a maior proporção de rebanhos positivos foi encontrada nesse circuito, com $21,0 \%$ de prevalência.

A análise das medidas obrigatórias estabelecidas pelo PNCEBT, como a vacinação, permite a avaliação de sua eficácia e impacto na redução da prevalência da enfermidade. Segundo Guimarães (2011), a cobertura vacinal contra a brucelose no Brasil cresceu gradativamente de 2001 para 2010. Apesar de ser um resultado satisfatório, o autor identificou que o número de fêmeas em idade vacinal foi superestimado devido a falhas nos registros. Dessa forma, a análise de dados permite avaliar possíveis falhas nos programas de controle, além de gerar informações de relevância, como por exemplo, para quais áreas os recursos devem ser destinados.

Dessa forma, o objetivo do trabalho foi analisar a tendência de longo prazo das taxas de vacinação no Sudoeste goiano, no período de 2015 a 2020.

\section{Metodologia}

Foi conduzido um estudo com análise de série temporal com base no número total de fêmeas entre três e oito meses de idade no Sudoeste Goiano, no período de 2015 a 2020. Os dados foram obtidos por meio de contato com a Agência Goiana de Defesa Agropecuária (Agrodefesa) via email. Foram utilizados exclusivamente dados provenientes de arquivos fornecidos pela Agrodefesa. Portanto, o estudo foi dispensado do comitê de ética pelo CEUA da Universidade Federal de Jataí.

Para realização da pesquisa, utilizou-se dados da microrregião definida pelo Instituto Brasileiro de Geografia e Estatística (Instituto Brasileiro de Geografia e Estatística [IBGE], 2014) como Sudoeste de Goiás entre o primeiro semestre de 2015 e primeiro semestre de 2020 que foram agrupados a partir das informações dos seus 18 municípios. O ano de 2016 foi excluído da análise devido à ausência de relatórios semestrais, pois nesse ano a Agrodefesa elaborou apenas um relatório anual de vacinação do rebanho bovino.

A taxa de vacinação de bovinos, expressa em porcentagem, foi calculada pela relação entre o número de fêmeas bovinas vacinadas e o número total de fêmeas bovinas entre três e oito meses de idade. Essa taxa foi calculada por semestre, pois o PNCEBT obriga o produtor a informar, o número de bezerras vacinadas e o número de bezerras existentes, em maio e em novembro de cada ano.

A análise da tendência de longo prazo de variação das taxas de vacinação realizada nesse estudo foi baseada no trabalho de Cruz (2018), utilizando-se a análise de tendência por meio da regressão de Prais-Winsten, que leva em consideração a autocorrelação serial (Wooldridge, 2013). A partir das taxas de incremento semestral (TIS), intervalos de confiança de 95\% (IC95\%) e valores de P (nível de significância de 5\%), classificou-se a tendência das taxas como crescente, estável ou decrescente. Valores de P não significativos resultaram em tendência de estabilidade (aceitando-se a hipótese nula de que as taxas analisadas não se modificaram significativamente ao longo do tempo). Valores de $\mathrm{P}$ significativos, resultaram em classificação de tendência de crescimento (TIS positiva) e tendência de diminuição (TIS negativa) (Cruz, 2018). A análise de regressão de Prais-Winsten foi realizada utilizando-se o pacote "Prais" do software R, versão 4.0.3 ${ }^{\text {. }}$

Para comparação global das taxas de vacinação dos municípios em cada semestre, realizou-se o teste do qui-quadrado, utilizando o pacote "binom" do software R. A partir dos limites inferiores e superiores, comparou-se cada município entre o primeiro e segundo semestres de cada ano, no período de 2015 a 2019. A sobreposição dos intervalos, revelou uma diferença não significativa ( $\mathrm{p}>0,05)$, e a não sobreposição, uma diferença significativa $(\mathrm{p}<0,05)$. 


\section{Resultados}

A partir da população de fêmeas bovinas em idade de vacinação e fêmeas bovinas vacinadas no Sudoeste Goiano, foram calculadas, entre os anos de 2015 a 2020, as taxas semestrais de vacinação (Tabela 1).

Ao analisar as taxas de vacinação por município, nota-se que Santa Rita do Araguaia (81\%) e Castelândia (81\%) se destacaram por apresentar as maiores taxas de vacinação no primeiro semestre de 2015. No segundo semestre do mesmo ano, Aporé (48\%) apresentou a taxa mais elevada. Já no primeiro semestre de 2017, Santa Rita do Araguaia (92\%) se destacou novamente com a maior taxa, no entanto, no segundo semestre de 2017, Jatai (39\%), obteve a taxa mais alta. Em 2018, no primeiro semestre, Castelândia apresentou taxa de vacinação de $71 \%$, sendo a maior desse período. No segundo semestre de 2018, Perolândia (45\%) demonstrou a maior taxa de vacinação. Por fim, no primeiro semestre de 2019 e de 2020, Santa Rita do Araguaia foi o município com as maiores taxas, com $81 \%$ e $117 \%$, respectivamente. No segundo semestre de 2019, Maurilândia obteve destaque ao apresentar taxa de vacinação de 51\%.

Quanto às taxas de vacinação mais baixas, Palestina de Goiás (39\%) destaca-se no primeiro semestre de 2015, no entanto, no segundo semestre do mesmo ano, podemos salientar a taxa observada no município de Chapadão do Céu (19\%). Em 2017, no primeiro semestre, Santa Helena de Goiás (37\%) e Maurilândia (37\%), apresentaram as menores taxas de vacinação. No segundo semestre de 2017, Chapadão do Céu voltou a se destacar ao apresentar uma taxa de 8\%. Santa Helena de Goiás (29\%), no primeiro semestre de 2018 apresentou a menor taxa entre os municípios. Já no segundo semestre de 2018, Aporé (9\%) obteve a menor taxa. Finalmente, no primeiro semestre de 2019 e de 2020, Chapadão do Céu, apresentou respectivamente, taxas de $32 \%$ e $28 \%$. O segundo semestre de 2019 foi marcado pela taxa apresentada pelo município de Aporé (12\%).

O município de Perolândia com taxa de variação de $13,86 \%$ (IC $95 \%$ 1,2\% a 28,1\%), apresentou diferença significativa quando comparado aos municípios de Aporé - 23,4\% (IC 95\% - 37,2\% a - 6,8\%), Chapadão do Céu - 15,1\% (IC 95\% - 28,7\% a 1\%), Palestina de Goiás - 5,1\% (IC 95\% -7,6\% a - 2,7\%), Portelândia 2,2\% (IC 95\% - 15,4\% a 23,5\%), Santa Helena de Goiás 8,4\% (IC 95\% - 14,7\% a - 1,9\%), e Serranópolis - 9,5\% (IC 95\% - 17,1\% a - 1,3\%). As demais comparações entre os municípios, não apresentaram diferença significativa.

No teste do qui-quadrado, após comparação global de todos os semestres, a diferença entre as taxas de vacinação dos diversos municípios é altamente significativa, com valor de $\mathrm{P}$ semelhante a zero. Comparando o mesmo município entre o primeiro e segundo semestres de cada ano, observou-se que em 2015 e 2017, somente Santa Helena de Goiás não apresentou diferença significativa. No ano de 2018, Perolândia foi o único município a apresentar uma diferença não significativa. Por fim, em 2019, todos os municípios apresentaram uma diferença altamente significativa. 
Tabela 1. Taxa semestral de vacinação contra brucelose bovina no período de 2015 a 2020, no Sudoeste Goiano.

\begin{tabular}{|c|c|c|c|c|c|c|c|c|c|c|c|c|c|}
\hline Município & $1^{\circ} / 2015$ & $2^{\circ} / 2015$ & $1 \% / 2017$ & $2^{\circ} / 2017$ & $1^{\circ} / 2018$ & $2^{\circ} / 2018$ & $1^{\circ} / 2019$ & $2^{\circ} / 2019$ & $1^{\circ} / 2020$ & $\begin{array}{l}\text { Média } \\
(\%)\end{array}$ & $\begin{array}{c}\text { Taxa de variação } \\
\text { Semestral } \\
\text { (IC95\%) }\end{array}$ & $\mathrm{p}-$ valor $^{\mathrm{a}}$ & Situação \\
\hline Ap. do Rio Doce & $63 \%$ & $36 \%$ & $56 \%$ & $29 \%$ & $50 \%$ & $22 \%$ & $65 \%$ & $30 \%$ & $46 \%$ & $44 \%$ & $-5,82$ & 0,0003023 & estável \\
\hline Aporé & $75 \%$ & $48 \%$ & $49 \%$ & $30 \%$ & $42 \%$ & $9 \%$ & $66 \%$ & $12 \%$ & $69 \%$ & $44 \%$ & $-23,49$ & 0,001108 & decrescente \\
\hline Caiapônia & $57 \%$ & $34 \%$ & $55 \%$ & $27 \%$ & $51 \%$ & $25 \%$ & $63 \%$ & $28 \%$ & $58 \%$ & $44 \%$ & $-1,92$ & $1,31 \mathrm{E}-02$ & estável \\
\hline Chapadão do Céu & $75 \%$ & $19 \%$ & $64 \%$ & $8 \%$ & $49 \%$ & $20 \%$ & $32 \%$ & $15 \%$ & $28 \%$ & $34 \%$ & $-15,13$ & 0,0006633 & estável \\
\hline Doverlândia & $67 \%$ & $35 \%$ & $58 \%$ & $32 \%$ & $52 \%$ & $26 \%$ & $70 \%$ & $33 \%$ & $67 \%$ & $49 \%$ & $-0,04$ & 0,0003533 & estável \\
\hline Jataí & $51 \%$ & $33 \%$ & $53 \%$ & $39 \%$ & $46 \%$ & $25 \%$ & $69 \%$ & $27 \%$ & $61 \%$ & $45 \%$ & $-1,07$ & $5,60 \mathrm{E}-02$ & estável \\
\hline Maurilândia & $49 \%$ & $29 \%$ & $37 \%$ & $23 \%$ & $42 \%$ & $33 \%$ & $43 \%$ & $51 \%$ & $60 \%$ & $41 \%$ & 13,96 & 0,0431 & estável \\
\hline Mineiros & $67 \%$ & $43 \%$ & $58 \%$ & $36 \%$ & $62 \%$ & $38 \%$ & $71 \%$ & $32 \%$ & $64 \%$ & $52 \%$ & $-2,74$ & 7,97E-03 & estável \\
\hline Montividiu & $52 \%$ & $28 \%$ & $47 \%$ & $32 \%$ & $46 \%$ & $22 \%$ & $58 \%$ & $30 \%$ & $38 \%$ & $39 \%$ & $-1,87$ & $8,96 \mathrm{E}-02$ & estável \\
\hline Palestina de Goiás & $39 \%$ & $33 \%$ & $41 \%$ & $25 \%$ & $43 \%$ & $24 \%$ & $44 \%$ & $21 \%$ & $48 \%$ & $35 \%$ & $-5,19$ & 0,002781 & decrescente \\
\hline Portelândia & $61 \%$ & $46 \%$ & $57 \%$ & $32 \%$ & $45 \%$ & $26 \%$ & $67 \%$ & $47 \%$ & $68 \%$ & $50 \%$ & 2,24 & 0,1868 & estável \\
\hline Rio Verde & $43 \%$ & $40 \%$ & $46 \%$ & $30 \%$ & $41 \%$ & $26 \%$ & $52 \%$ & $24 \%$ & $45 \%$ & $39 \%$ & $-7,16$ & 0,01778 & decrescente \\
\hline Santa Helena & $40 \%$ & $39 \%$ & $37 \%$ & $38 \%$ & $29 \%$ & $26 \%$ & $39 \%$ & $25 \%$ & $38 \%$ & $35 \%$ & $-8,47$ & $5,99 \mathrm{E}-02$ & decrescente \\
\hline Santa Rita & $81 \%$ & $32 \%$ & $92 \%$ & $19 \%$ & $64 \%$ & $33 \%$ & $81 \%$ & $14 \%$ & $117 \%$ & $59 \%$ & $-8,57$ & 0,1717 & decrescente \\
\hline Santo Ant. da Barra & $47 \%$ & $33 \%$ & $58 \%$ & $36 \%$ & $41 \%$ & $17 \%$ & $52 \%$ & $27 \%$ & $59 \%$ & $41 \%$ & $-5,50$ & 0,01142 & decrescente \\
\hline Serranópolis & $55 \%$ & $37 \%$ & $55 \%$ & $29 \%$ & $42 \%$ & $21 \%$ & $63 \%$ & $20 \%$ & $58 \%$ & $42 \%$ & $-9,53$ & 0,03529 & decrescente \\
\hline
\end{tabular}

Fonte: Pesquisa Própria. 


\section{Discussão}

Com base no modelo de Amaku et al. (2009), desenvolvido para o Estado de São Paulo, altas coberturas vacinais (acima de 70\%) são eficazes no controle da brucelose bovina, levando menos tempo para atingir uma taxa de prevalência de $2 \%$, do que quando comparado com baixas coberturas vacinais (inferiores a 30\%). Para regiões com taxas de vacinação elevadas, o estudo prevê um período de 10 anos para tal redução de prevalência. O dobro desse período seria necessário para locais com baixas coberturas vacinais. Minervino et al. (2011), em seu estudo no Estado do Pará, conseguiram evidenciar a influência da vacinação na prevalência da brucelose, revelando um maior número de casos nos rebanhos não vacinados do que naqueles cuja vacinação é feita nas fêmeas de três a oito meses.

Uma vez que, não foram realizados no Estado de Goiás estudos utilizando modelos matemáticos, optou-se por usar como base, pesquisas realizadas em outros estados para se traçar uma tendência do que poderia acontecer com relação à microrregião do Sudoeste Goiano, que tem como média vacinal 44\%. A partir dos estudos supracitados, observa-se que não haveria redução na prevalência da brucelose bovina no sudoeste goiano, pois as taxas de vacinação estão baixas. O estado de São Paulo, que nos últimos 10 anos obteve uma média das taxas de vacinação acima de 80\%, é um dos exemplos de sucesso do programa de vacinação contra a brucelose (Cruz, 2018).

Foi observada tendência de estabilidade das taxas de vacinação em 10 dos 18 municípios do Sudoeste Goiano; o que seria um resultado satisfatório caso as taxas estivessem acima de 70\%, levando-se em conta o trabalho de Amaku et al. (2009). Por isso, acredita-se que os municípios em questão levarão mais tempo para reduzir a prevalência da brucelose bovina, de $3 \%$ (Rocha et al., 2009) para $2 \%$.

Apenas o município de Perolândia apresentou tendência de crescimento das taxas de vacinação. Apesar de ser um resultado positivo, as taxas em todos os semestres foram inferiores a 70\%, revelando uma oscilação acentuada entre o primeiro e segundo semestres de cada ano. Dessa forma, mesmo com a tendência de crescimento, esse resultado não reflete evolução no programa de vacinação contra a brucelose bovina na região. De acordo com Nobre (2016), a contratação de fiscais agropecuários, juntamente com a educação sanitária, visando o aumento da conscientização dos proprietários sobre a importância da imunização, pode contribuir para o aumento dos índices vacinais. Os demais municípios apresentaram tendência de diminuição das taxas de vacinação, refletindo uma situação preocupante, pois além das taxas já estarem baixas, a tendência é diminuir ainda mais com o passar o tempo.

Em todo o período avaliado, a Agrodefesa registrou no $1^{\mathrm{o}}$ e $2^{\mathrm{o}}$ semestres de cada ano, o mesmo número total de fêmeas de 3 a 8 meses (idade de vacinação); o que pode ter prejudicado a análise, influenciando nas baixas taxas de vacinação observadas em todos os $2^{\circ}$ semestres, pois muitas das bezerras passíveis de vacinação no primeiro semestre podem ter passado da idade no segundo semestre, e mesmo assim, estão sendo consideradas nos cálculos das taxas.

Santa Rita do Araguaia no $1^{\circ}$ semestre de 2020 apresentou uma taxa de vacinação superior a 100\% (117\%). Tal resultado pode estar relacionado à subnotificação e em como os dados são armazenados. A utilização de um sistema informatizado, como o sistema de Gestão de Defesa Animal e Vegetal (GEDAVE) empregado no Estado de São Paulo, segundo Cruz (2018), é uma forma de gerar dados confiáveis para estudos epidemiológicos, além de auxiliar no desenvolvimento do PNCEBT. Nesse banco de dados, são registradas informações a respeito de vacinação, propriedades com bovinos reagentes, número de bovinos reagentes e número de exames realizados. Conforme Cruz. (2018), a implementação dessa plataforma levou a homogeneização dos dados. Sendo assim, existe a necessidade do estabelecimento de um sistema para registro dos dados no estado de Goiás, de forma a aumentar a qualidade das análises.

Nos relatórios mensais e semestrais compartilhados pela Agrodefesa, fêmeas bovinas de 0 a 12 meses são consideradas fêmeas em idade vacinal. Dessa forma, fêmeas adultas podem estar sendo vacinadas com a amostra B19. De 
acordo com Brasil (2006), quando se vacina fêmeas com mais de oito meses de idade com a B19, ocorre interferência no diagnóstico sorológico, acarretando em resultados falsos positivos. A administração após os oito meses, induz a formação de anticorpos específicos, que persistirão após os 24 meses, culminando em falhas no diagnóstico da enfermidade. A vacina quando realizada no período recomendado, não gera falsos resultados, pois os anticorpos desaparecem antes da fêmea completar 24 meses. Para vacas com mais de oito meses, o PNCEBT preconiza o uso de uma cepa rugosa (RB51) de Brucela abortus, não indutora de anticorpos aglutinantes no teste sorológico padrão para brucelose bovina (Brasil, 2006). Além do uso indevido da B19 em fêmeas acima de 8 meses, existe a possibilidade de fêmeas com menos de 3 meses também estarem sendo vacinadas com a B19. A utilização da cepa B19 nessa faixa etária não é recomendada, pois o sistema imunológico das bezerras ainda não está apto a induzir uma resposta imune efetiva (Paulin \& Ferreira Neto, 2003).

Segundo dados de Ferreira Neto et al. (2016), a produção da amostra vacinal B19 por laboratórios particulares, desde o estabelecimento do PNCEBT até 2012, sofreu um aumento significativo. Já a produção da amostra RB51, desde sua liberação, sofreu uma diminuição no decorrer do tempo. O declínio na produção da cepa RB51 pode estar relacionado a baixa aceitação por parte dos produtores, explicada pela falta de conhecimento sobre o papel e importância dessa vacina no contexto do PNCEBT, uma vez que sua aplicação não se limita pela idade da fêmea, além de não interferir nos testes diagnósticos da brucelose bovina, evitando resultados falsos positivos e consequente redução do número de casos. Analisar a cobertura vacinal da cepa RB51 no estado de Goiás é uma forma de complementar o presente estudo e auxiliar na elaboração de novas estratégias de controle pelo MAPA.

A diferença altamente significativa das taxas de vacinação observadas entre os municípios pode estar relacionada á dados deficientes. Segundo Guimarães (2011), a padronização do envio de relatórios, de forma a unificar o formato dos arquivos (Excel, Word), é um meio de facilitar a compilação dos dados e reduzir os erros durante a sua coleta, aumentando assim, a qualidade das informações. Outro fator relacionado a qualidade dos dados é a ausência de um sistema de informação informatizado, uma vez que as informações não podem ser conferidas e atualizadas de forma rápida e constante (Guimarães, 2011). Cruz (2018), identificou uma grande oscilação dos valores anuais das taxas de vacinação no Estado de São Paulo antes da implementação da plataforma GEDAVE. Dados mais homogêneos foram observados após seu estabelecimento, demonstrando a influência da informatização na qualidade das informações.

\section{Conclusão}

As taxas de vacinação no Sudoeste Goiano não são suficientes para que a prevalência da brucelose seja reduzida. Portanto, deve-se buscar atingir taxas superiores a 80\% para tal redução. Ampliar a aceitação da cepa RB51 é uma forma de aumentar a cobertura vacinal, uma vez que pode ser administrada em fêmeas adultas. Dessa forma, a criação de um programa de orientação para produtores pelo PNCEBT se mostra necessário.

\section{Referências}

Amaku, M., Dias, R. A., Ferreira Neto, J. S., \& Ferreira, F. (2009). Modelagem matemática do controle de brucelose bovina por vacinação. Arquivo Brasileiro de Medicina Veterinária e Zootecnia, 61 (1). https://doi.org/10.1590/S0102-09352009000700017

Brasil. (2006). Programa Nacional de Controle e Erradicação da Brucelose e da Tuberculose Animal. Ministério da Agricultura, Pecuária e Abastecimento. http://www.saudeanimalms.com.br/files/servicos/manual-pncebt_38562454.pdf

Brasil. (2017). Instrução Normativa $n^{o}$ 10, de 3 de março de 2017. Ministério da Agricultura, Pecuária e Abastecimento. https://www.in.gov.br/materia//asset_publisher/Kujrw0TZC2Mb/content/id/19124587/do1-2017\%E2\%80\%9306-20instrucao-normativa-n-10-de-3-de-marco-de-017\%E2\%80\%9319124353

Brasil. (1977). Diagnóstico de saúde animal, Brasília. Ministério da Agricultura e Abastecimento.

Cruz, C. A. (2018). Indicadores relacionados à evolução do Programa de Controle e Erradicação da Brucelose Bovina no Estado de São Paulo [Tese de doutorado, Universidade Estadual Paulista]. RI UNESP. https://repositorio.unesp.br/bitstream/handle/11449/180931/cruz_ca_dr_jabo_int.pdf?sequence=5 
Research, Society and Development, v. 10, n. 7, e40710716685, 2021

(CC BY 4.0) | ISSN 2525-3409 | DOI: http://dx.doi.org/10.33448/rsd-v10i7.16685

Ferreira Neto, J. S., Silveira, G. B., Rosa, B. M., Gonçalves, V. S. P., Grisi-Filho, J. H. H., Amaku, M., Dias, R. A., Ferreira, F., Heinemann, M. B., Telles, E. O., \& Lage, A. P. (2016). Semina: Ciências Agrárias, 37 (5), 3385-3402. http://dx.doi.org/10.5433/1679-0359.2016v37n5Sup12p3385

Guimarães, G. O. (2011). Programa Nacional de Controle e Erradicação de Brucelose e Tuberculose Animal (PNCEBT): evolução no controle da brucelose bovina de 2001 a 2010 [Monografia, Universidade de Brasília]. BDM UNB. https://bdm.unb.br/handle/10483/3051

Instituto Brasileiro de Geografia e Estatística. (2014). [Mapa] Microrregião do IBGE do Sudoeste de Goiás. https://www.imb.go.gov.br/files/docs/mapas/microrregioes-ibge/microrregiao_do_sudoeste_de_goias.pdf

Leal Filho, J. M., Bottene, I. F. N., Monteiro, L. A. R. C. M., Pellegrin, A. O., Gonçalves, V. S. P., Ferreira, F., Dias, R. A., Amaku, M., Telles, E. O., GrisiFilho, J. H. H., Heinemann, M. B., \& Ferreira Neto, J. S. (2016). Control of bovine brucellosis from 1998 to 2009 in the state of Mato Grosso do Sul, Brazil. Semina: Ciências Agrárias, 37 (5), 3467-3478. https://doi.org/10.5433/1679-0359.2016v37n5Supl2p3467

Megid, J., Ribeiro, M. G., \& Paes, A. C. (2016). Doenças Infecciosas em Animais de Produção e Companhia. ROCA.

Minervino, A. H. H., Antonio, A. S., Alves-Filho, A., Barbosa, R. S., Neves, K. A. L., Barros, I. O., Barreto, R. A., \& Ortolani, E. L. (2011). Acta Veterinaria Brasilica, 5 (1), 47-53. https://doi.org/10.21708/avb.2011.5.1.1807

Nobre, A. F., Barros-Neto, S., Galindo, G. A. R., Rodrigues, F. C., Girardeli, G. L., Alves, A. J. S., Marinho, M., \& Queiroz, L. H. (2016). Evolução das taxas de vacinação contra brucelose bovina segundo as áreas de risco para febre aftosa no Estado do Pará. Veterinária e Zootecnia, 23(3), 483494.https://www.researchgate.net/publication/308084616_EVOLUCAO_DAS_TAXAS_DE_VACINACAO_CONTRA_BRUCELOSE_BOVINA_SEGUND O_AS_AREAS_DE_RISCO_PARA_FEBRE_AFTOSA_NO_ESTADO_DO_PARA

Paulin, L. M., Ferreira Neto, J. S. (2003). O combate à brucelose bovina: Situação brasileira. FUNEP.

Poester, F., Figueiredo, V. C. F., Lôbo, J. R., Gonçalves, V. S. P., Lage, A. P., Roxo, E., Mota, P. M. P. C., Müller, E. E., \& Ferreira Neto, J. S. (2009). Analysis of 15 years of the National Program for the Control and Eradication of Animal Brucellosis and Tuberculosis, Brazil. Arquivo Brasileiro de Medicina Veterinária e Zootecnia, 61 (1). https://doi.org/10.1590/S0102-09352009000700001

Santana, D. C., \& Veiga, L. S. (1982). Brucelose bovina em Goiás.

Santos, A. J. F. (2017). Soroconversão atribuível à vacinação de bezerras com a CEPA B-19 de Brucella abortus [Dissertação de mestrado, Universidade Federal de Tocantins]. RI UFT. http://200.129.179.47/bitstream/11612/855/1/Alessandro\%20Jos\%c3\%a9\%20Ferreira\%20dos\%20Santos\%20$\% 20$ Disserta\%c3\%a7\%c3\%a3o.pdf

Ragan, V. E. (2002). The Animal and Plant Health Inspection Service (APHIS) brucellosis eradication program in the United States. Veterinary Microbiology, 90 (1-4), 11-8. https://doi.org/10.1016/s0378-1135(02)00240-7

Silva, T. I. B., Moraes, R. S., Santos, P. S., Reckziege, G. R., Gomes, Y. A., Melchior, L. A. K., Fernandes, A. C. C., Baptista-Filho, L. C. F., Silva, D. D., Revoredo, R. G., \& Melo, L. E. H. (2019). Analysis of the risk factors for bovine brucellosis in dairy herds of the Rio Branco microregion, Acre, Brazil. Arquivos do Instituto Biológico. 86, 1-6. https://doi.org/10.1590/1808-1657000792018

Wooldridge, J. M. (2013). Introductory econometrics: a modern approach. Mason. Cengage Learning. 